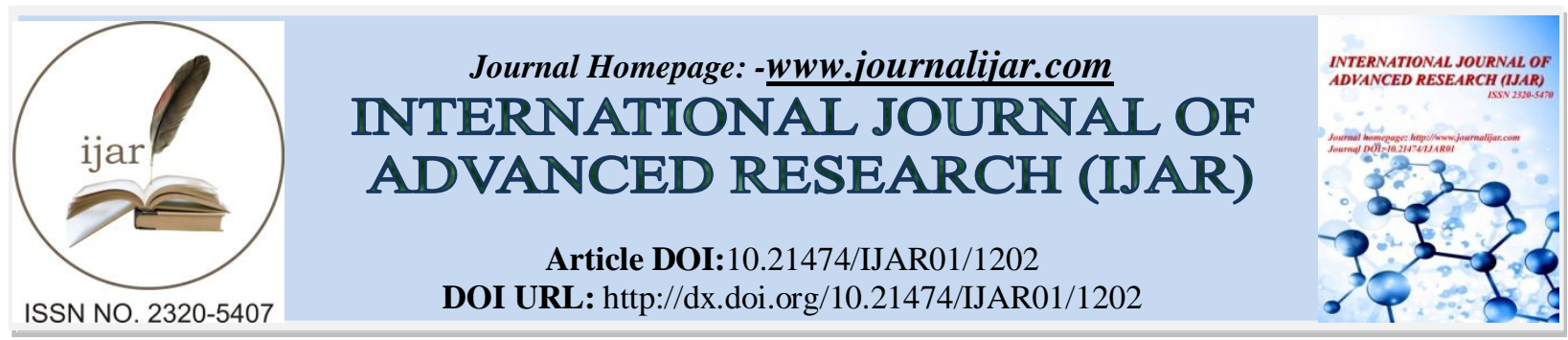

RESEARCH ARTICLE

\title{
FORMATION OF HIGH TEMPERATURE CORROSION PRODUCTS OF AISI 310S AUSTENITIC STAINLESS STEEL.
}

Tosapolporn Pornpibunsompop ${ }^{1,2}$ and Purit Thanakijkasem ${ }^{2}$.

1. Metallurgical and material engineering laboratory, Defense technology institute, THAILAND.

2. Division of Materials Technology, School of Energy, Environment and Materials, KMUTT, THAILAND

\section{Manuscript Info}

-

Manuscript History

Received: 16 June 2016

Final Accepted: 16 July 2016

Published: August 2016

Key words:-

High temperature corrosion, Austenitic stainless steel, Corrosion products.

\begin{abstract}
The Formation of high temperature corrosion products in chloride containing environment was investigated by optical microscopy. Chloride environment originated from chemically preparation of hydrochloric solution, then boiled it until meets boiling point, and finally fed through nozzle into horizontal chamber, which was heated up from ambient to $900^{\circ} \mathrm{C}$, in term of acidic vapor. The test results obtained that chromium carbide and sigma phase precipitates at grain boundaries and within austenite matrix. The formation of chromium carbide and sigma phase are mutually complex reaction. The precipitation effects to generate chromium depleted zone and would lead to intergranular corrosion. The high temperaturechloride containing environment results in oxide and chloride corrosion products on stainless steel surface. Moreover, wet condition deteriorates to chromium oxide film and produces ferrous oxide combined with chloride metalliccompounds.
\end{abstract}

Copy Right, IJAR, 2016,. All rights reserved.

\section{Introduction:-}

[1-2]High temperature corrosion of austenitic stainless steel was examined with severalresearchers because it was widely existed in many industries for example power generation, aerospace, gas turbine, automotive, defense etc. A well-known phenomenon athigh temperature is oxidation which its properties such astheir thermodynamic stability, ionic defect structure, and detailed morphology,play a crucial role in determining the oxidation resistance of stainless steel in a specific environment. The appropriate quantity of chromium is a main cause to improve corrosion resistance of stainless steel in a number of environments because it, at least $11 \%$ of $\mathrm{Cr}$, leads to exhibit passive characteristic by generation of chromium oxide film. The methods would enhance performance of high-temperature oxide filmare increase quantity of chromium or increase content of aluminum or silicon in order to alternatively form more stabilized passive oxides at high temperature. In many industries, oxidation does not independently occur; rather a combination of high-temperature corrosion caused by contaminants for example chloride, sulfur, humidity etc. are present in the atmosphere. Those contaminants would accelerate high temperature corrosion due to have a strong drawback synergistic effect with oxidation. Chlorides contribute to high-temperaturecorrosion by interfering with the formation of protective oxides orbreaking them down if already formed. When both chlorine and oxygen are presentin the environment, essentially a competing situation arises betweenthe formation of stable oxide and volatile chloride products. The latter component is a crucial reason for corrosion andthe degradation rate cannot be precisely predicted.Not only the composition of corrosive environment, but occurring of metallurgical phase at

Corresponding Author:-TosapolpornPornpibunsompop

Address:-Metallurgical and material engineering laboratory, Defense technology institute, THAILAND. 
elevated temperature also mutually deteriorate on austenitic stainless steel especially high alloying addition grades. With existing of precipitated phases (such as sigma, Laves, and Chi phases), the ductility and toughness at room temperature are significantly poor. The research of high temperature corrosion of austenitic stainless steel would still carry out continuously in order to investigate and conclude the best solution for each new combination of stainless steel-corrosion environment in the future.

[3] studiedthe effect of $\mathrm{CaCl}_{2}$ and $\mathrm{BaCl}_{2}$ salt coatings on the high temperature corrosion of 321 stainless steel at 950 ${ }^{\circ} \mathrm{C}$ in a slow currentof air for the period of 72 hours. The alloy was severely attacked by the formation of volatile chlorides. The alkaline earth metal chloride salts have detrimental effect on the protective capability of the scale and rapid degradation of the alloy. The trend of corrosion rate was identified by pattern of graph and concludes that when the temperature increases in the time for the initiatingcorrosion attack is decreased. [4]reviewed the high temperature chloride corrosion of metals and alloys at temperature more than 200 degree Celsius and concluded that high temperature corrosion consists mainly of the diffusion of ions in the scale and the volatilization of the scale. [45]the effect of water vapor on the corrosion resistance of most metals might beneficial due to the formation of a protective oxide in contrast with Cr-containing steel. It is detrimental to corrosion resistance of Cr-containing steel. Additionally, the research on precipitation of metallic compounds in stainless steel was studied by [6-7]. Intense of intermetallic compounds were conditionally formed when exposed temperature and incubation time were optimally reached. The compounds were detected in this study are carbides and sigma phase which become more intense with increase of temperature and time.

The objective of this paper was to characterize and discuss corrosion products building on and in austenitic stainless steel surface.

\section{Details experimental:-}

\section{Material:-}

The plate shape of wrought AISI 310 S austenitic stainless steel was selected for this investigation. The cut size of specimen, shown on Fig.1,is $140 \mathrm{~mm} \times 30 \mathrm{~mm} \times 3 \mathrm{~mm}(\mathrm{~L} \times \mathrm{W} \times \mathrm{t})$. Table 1 displays chemical composition of SUS $310 \mathrm{~S}$ supplied by domestic supplier. When compares among others type of austenitic stainless steel, for example 304 and 316, 310S contains carbon, chromium, and nickel higher than 304 and 316 stainless steel. The main objective is to enhance corrosion resistance capability and increase working temperature. The specimens were bend into U-shape and then polished at curvature surface to remove scales and contaminants.

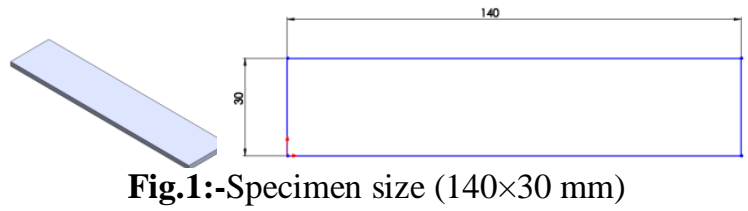

Table1:-Chemical Composition of SUS310S austenitic stainless steel.

\begin{tabular}{|l|c|c|c|c|c|}
\hline Element & $\mathrm{C}$ & $\mathrm{Cr}$ & $\mathrm{Ni}$ & $\mathrm{Si}$ & $\mathrm{Mn}$ \\
\hline Quantity (\%) & 0.08 & 24.58 & 19.13 & 0.59 & 1.85 \\
\hline
\end{tabular}

High temperature corrosion test:-

The high temperature corrosion test (Fig.2) was carried out within horizontal ceramic tube chamber. It can digitally control heating cycle and can feed intended gas or liquid into the chamberthrough three nozzles in order to create a corrosive atmosphere.

The solution was prepared by mixing between $\mathrm{HCl}$ solution and distilled water with ratio $6: 1$ by mole. Additionally, the solution was boiled until generates vapor. Vapor can feed through silicone hose and nozzle into the chamber depending upon vapor pressure of $\mathrm{HCl}$ solution and creates wet acidic chloride corrosive environment within chamber.

In this study, the chamber heats up from ambient to $900^{\circ} \mathrm{C}$ about 4 hours, holds on 120 hours, and cools down to ambient temperature according to Fig.3. The acidic vapor was fed and kept within closed chamber for 120 hours. 
The corrosion reaction and oxidation would occur simultaneously forming corrosion products on specimen surface and crack site.

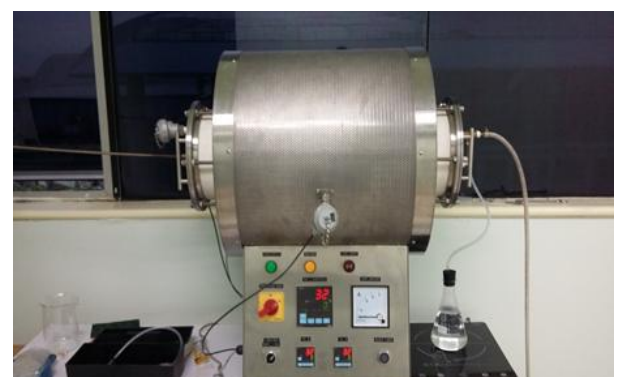

Fig.2:-Corrosion testing chamber.

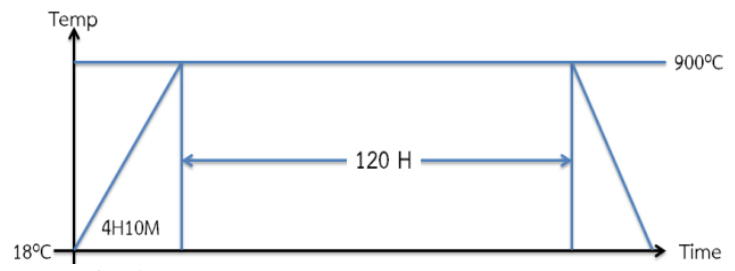

Fig.3:-Heating cycle for corrosion testing.

\section{Non-destructive testing for crack detection:-}

Radiographic inspection by x-ray uses to monitor, inspect, and diagnostic material discontinuities at surface and subsurface. This method utilizes x-rays which arevery shortwavelength electromagnetic waves. The waves penetrate the material and are absorbed,depending on the thickness or the density of the material beingexamined. So that the thickness and type of studied material are important parameters to choose this method for inspection.In this research, $\mathrm{x}$-ray was used to detect some cracks which might be originated on specimen surface after passing corrosion test operation.

\section{Material characterization:-}

Microstructures were analyzed by light optical microscope with specimen prepared by mechanical polishing and finally etching in etchants.

The etchants used in this investigation shows on Table 2.

Table2:-Chemical Composition of used etchants.

\begin{tabular}{|c|c|c|c|}
\hline No. & Etchants & Composition & Method \\
\hline 1 & Kalling's reagent & $\begin{array}{c}5 \mathrm{grCuCl}_{2}+100 \mathrm{ccHCl}+100 \mathrm{ccC}_{2} \mathrm{H}_{6} \mathrm{O} \\
(\mathrm{ASTM} \mathrm{E} \mathrm{407)}\end{array}$ & Immersion \\
\hline 2 & Marble's reagent & $\begin{array}{c}4 \mathrm{gCuSO}_{4}+200 \mathrm{ccHCl}+200 \mathrm{ccH}_{2} \mathrm{O} \\
(\text { ASTM E 407) }\end{array}$ & Immersion \\
\hline
\end{tabular}

Kalling's and Marble's reagent will react with specimen surface at grain boundaries and reveal significant microstructures and precipitation phases of stainless steel.

\section{Results and Discussion:-}

\section{Crack detection by radiographic testing:-}

$\mathrm{X}$-Ray radiographic test was systematically selected for this crack detection because the expected crack size and the thickness of specimen are suitable for crack evaluation. Comparative among two categories of specimens with radiographic pictures displayed that the crack was found on the top of curvature on specimen no. 2 because bending process will create plastic tensile strain and consequent stress on curvature surface depending directly on bending radius. Specimen no. 2 was permanently bended with radius more than specimen no. 1 and more susceptible to tear with external tension by bending as well. 
Microstructure evolution and discussion:-

Specimen no.2 was mechanically cut with low heat generation technique at crack area identified by $\mathrm{x}$-ray radiography as shown on Fig.4. Cutting direction was perpendicular to crack geometryin order to conveniently evaluate and analysis corrosion product building on crack surface.

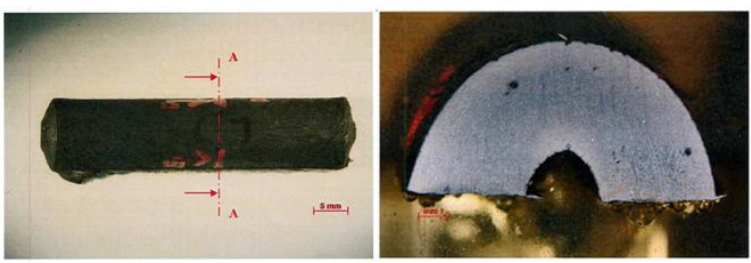

Fig.4:-Cutting surface at crack initiation area.

For primary investigation, the upper curvature surface was found some cracks. Fig.5 shows macroscopically investigates crack areas and could be divided into 2 main zones depending on crack position and possibility of corrosion product initiation. Zone A locates on the top of curvature surface and contacts directly to corrosive environment within testing chamber. Zone B locates on the side of specimen and contacts indirectly to corrosive environment.

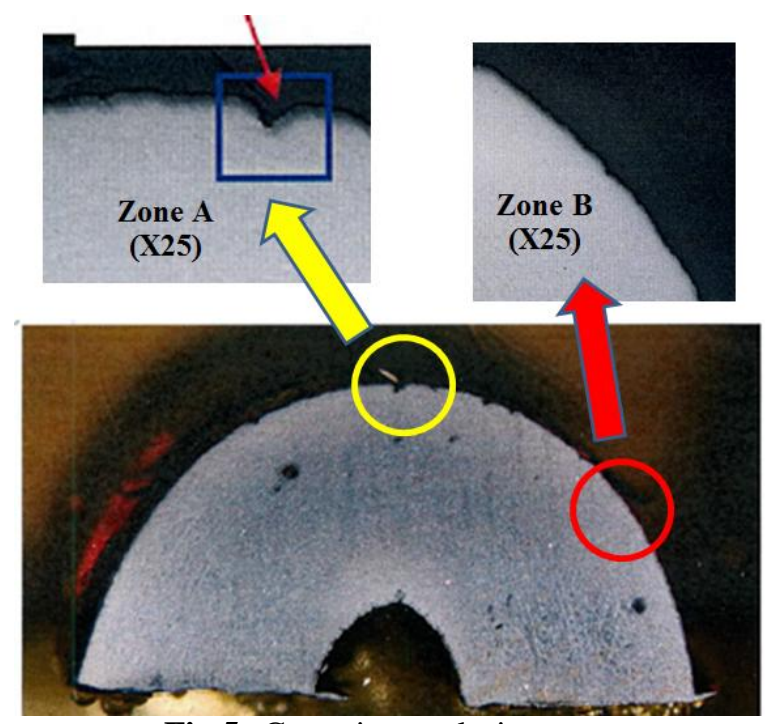

Fig.5:-Corrosion analysis zones.
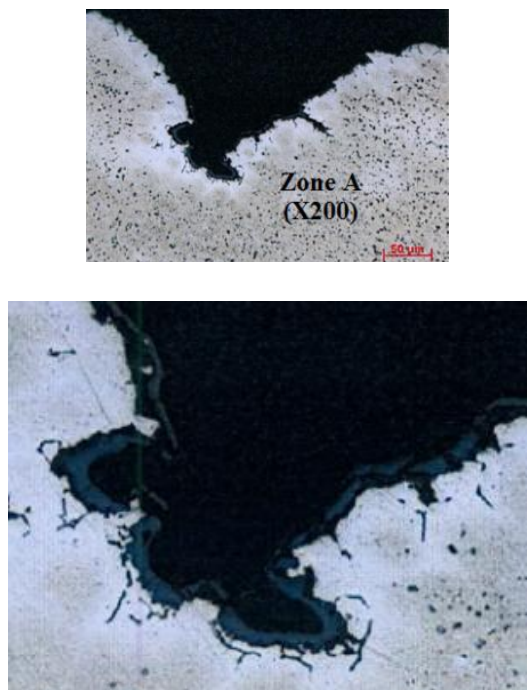

Fig.6:-Microstructure of zone A. 

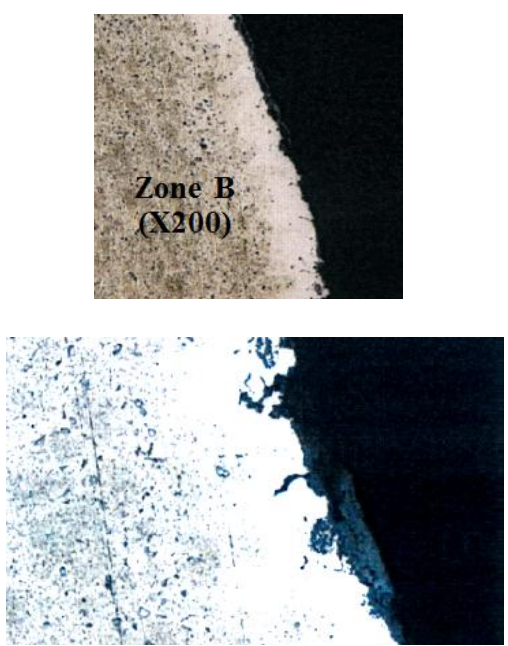

Fig.7:-Microstructure of zone B.

Fig.6-7investigation result revealed microstructure of zone A and zone B respectively and found some corrosion products on crack site. The corrosion products were constructed covering metal surface continuously in case of zone A in contrast with zone B that the corrosion products were intermittent built. The chromium depleted zone was identified along and near crack site in both zones. Additionally, chromium carbide $\left(\mathrm{Cr}_{23} \mathrm{C}_{6}\right)$ precipitation and sigma phase were also dispersedly formedon austenite matrix as shown on Fig.8.
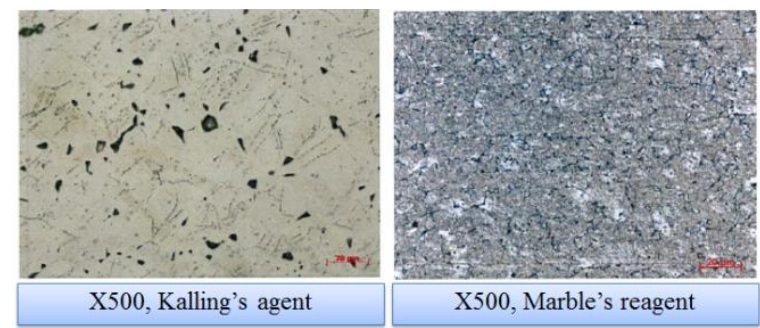

Fig.8:-Carbide arrangement in different etchants (Left: Kalling's agent, Right: Marble's reagent)

[1-2] The elevated temperature and wet chloride containing environment mutually accelerates corrosion product both on and in stainless steel surface. The temperature of surrounding exceeds $600^{\circ} \mathrm{C}$ will stimulate and lead tothe susceptibility to precipitation of chromium carbide $\left(\mathrm{Cr}_{23} \mathrm{C}_{6}\right)$ mostly at grain boundary. Additionally, the mechanism of sigma phase precipitation in austenitic stainless steels depends on residual chemical composition after chromium carbide precipitation.Sigma phase will precipitate within the matrix of austenite and both chromium carbide and sigma phase causes chromium depleted zone which is a sensitive area to intergranular corrosionoccurrence. For corrosive environment, wet condition (in term of $\mathrm{H}_{3} \mathrm{O}^{+}$)has been concentrated because it can destabilizeand attack chromium oxide layer which plays the important role to protect stainless steel surface from corrosion and originate ferrous oxide and chloride metallic compound forming. The formation sequence of ferrous oxide film considering from specimen's surface is Wütite $(\mathrm{FeO}) \rightarrow$ Magnetite $\left(\mathrm{Fe}_{3} \mathrm{O}_{4}\right) \rightarrow$ Hematite $\left(\mathrm{Fe}_{2} \mathrm{O}_{3}\right)$ are commonly observed. Their successive of such oxide compounds conform to the order of their free energies of formation and oxygen quantity. Wütite layer would contain compound of chloride (such as $\mathrm{FeCl}_{2}$ etc.).

\section{Conclusions:-}

The high temperature corrosion product formation of austenitic stainless steel was investigated and major conclusions are as follows:

1. At elevated temperature (exceed $600^{\circ} \mathrm{C}$ ), chromium carbide and sigma phase precipitate at grain boundary and within austenite matrix. The formation of chromium carbide and sigma phase are mutually complex reaction. The precipitation effects to chromium depleted zone and localized corrosion in the future. 
2. The high temperature corrosion in chloride containing environment results in oxide and chloride corrosion products on stainless steel surface. Wet condition severely destroys chromium oxide film and produces ferrous oxide $\left(\mathrm{Fe}_{\mathrm{x}} \mathrm{O}_{\mathrm{y}}\right)$ combined with chloride metallic compounds.

\section{Acknowledgments:-}

This research was fully supported by the Defense Technology Institute (Public Organization), Nonthaburi, Thailand. The financial support is gratefully acknowledged.

\section{References:-}

1. Robert, P.R. 2000. Handbook of Corrosion Engineering, $9^{\text {th }}$ ed., McGraw-Hill.

2. Lacombe, P. 1993. Stainless steels Volume 1\&2, Scientific ed., Ugine SAVOIE \& J and IMPHY S.A.

3. Amin, N., 2008, Therole of chloride salts on high temperature corrosion of 321 stainless steel, Materials and technology, vol.42, pp.273-276.

4. Chang, N., 1991, High temperature chlorine corrosion of metals and alloys, Material science, vol.26, pp.36933698.

5. Sorell, G., 1997, The role of chlorine in high temperature corrosion in waste-to-energy plants, Science and technology letters,0960-3409/97/03/00035-12, pp.137-150.

6. Tavares, S.S.M., 2008, Microstructural changes and corrosion resistance of AISI 310S steel exposed to 600$800^{\circ} \mathrm{C}$, Material characterization,vol.60, pp.573-578.

7. Wasnik, D.N., 2003, Precipitation stages in a 316L austenitic stainless steel, ScriptaMaterialia,vol.49, pp.135141. 